\title{
Efectos del Láser de Baja Intensidad (As-Ga-Al 904 nm) sobre el Cóndilo Mandibular de Conejos: Análisis a través de Tomografía Computadorizada Cone Beam
}

\author{
Effects of Low-Level Laser (Ga-Al-As $904 \mathrm{~nm}$ ) on the Mandibular \\ Condyle of Rabbits: Analysis by Cone Beam Computed Tomography
}

Nilton Alves*, Ivonne Garay ${ }^{* *} \&$ Naira Figueiredo Deana***

\begin{abstract}
ALVES, N.; GARAY, I. \& DEANA, N. F. Efectos del láser de baja intensidad (As-Ga-Al 904 nm) sobre el cóndilo mandibular de conejos: análisis a través de tomografía computarizada cone beam. Int. J. Morphol., 34(1):218-222, 2016.

RESUMEN: En la literatura científica los efectos adversos del láser de baja intensidad (LBI) no están descritos de forma precisa, tampoco su inocuidad. El objetivo de este estudio fue evaluar el efecto de distintas dosis de LBI en el cóndilo mandibular de conejos. Dieciséis conejos divididos aleatoriamente en cuatro grupos experimentales (GE) de 4 animales fueron utilizados en este estudio. Se realizó un examen de tomografía computarizada cone beam (TCCB) de ambas articulaciones temporomandibulares (ATMs) de todos los animales que fueron utilizadas como control (GC). Luego, irradiamos cada ATM con LBI (As-Ga-Al $904 \mathrm{~nm}$ ), $100 \mathrm{~mW}$, spot 0,2 cm², dos puntos, 3 veces/semana, totalizando 10 veces, con dosis de $15 \mathrm{~J} / \mathrm{cm}^{2}$ (GE-1), $45 \mathrm{~J} / \mathrm{cm}^{2}$ (GE-2), $60 \mathrm{~J} / \mathrm{cm}^{2}(\mathrm{GE}-3)$ y $90 \mathrm{~J} / \mathrm{cm}^{2}(\mathrm{GE}-4)$. Después de 20 días de la última irradiación se realizó un nuevo examen de TCCB y luego se obtuvo las mediciones de los cóndilos en los planos coronal y sagital. Para el análisis estadístico se utilizó las pruebas de ANOVA y t-student. Los valores promedios encontrados para el GE fueron mayores que los encontrados para el GC, en los planos coronal y sagital. Hubo diferencia estadística significativa para el grupo 1 y entre los grupos 1 y 2. Concluimos que los valores promedios encontrados en las mediciones de los cóndilos mandibulares, sometidos a las dosis de LBI utilizadas en este estudio, fueron mayores que los encontrados para el GC en los planos coronal y sagital. Además, la TCCB demostró ser un método eficaz para el análisis de las dimensiones condilares.
\end{abstract}

PALABRAS CLAVE: Tomografía computarizada cone-beam; Articulación temporomandibular; Conejo; Modelo animal; Láser de baja intensidad.

\section{INTRODUCCIÓN}

La articulación temporomandibular (ATM) en humanos es una articulación altamente especializada, clasificada como sinovial, bicondílea y triaxial. Sus superficies articulares están representadas por la vertiente posterior del tubérculo articular, porción anterior de la fosa mandibular del hueso temporal y vertiente anterior de la cabeza de la mandíbula o cóndilo de la mandíbula (Alves \& Cândido, 2013).

El conejo es un modelo animal adecuado para ser utilizado en el estudio de la ATM humana, ya que esta presenta similitudes morfológicas con la ATM de conejo y también los movimientos mandibulares durante la masticación son similares en conejos y humanos (Mills et al., 1994). Sin embargo, existen algunas diferencias entre la ATM humana y la de conejos. El hueso temporal de conejo presenta un proceso cigomático prominente que se articula con el hueso cigomático ventralmente. La depresión ubicada en la cara ventral del proceso cigomático forma la fosa mandibular, que se articula con el cóndilo de la mandíbula. Así la cara articular del hueso temporal se encuentra en el plano ventral del proceso cigomático del hueso temporal, es corta, estrecha y profunda. Otra diferencia es que el arco cigomático del conejo está formado por la fusión del proceso cigomático del maxilar y extremidad rostral del hueso cigomático. Caudodorsalmente el arco se articula con el proceso cigomático del temporal. No hay proceso retroarticular, que en humanos, es la estructura que protege la porción timpánica del temporal contra un desplazamiento exa-

* CIMA Research Group, Departamento de Odontología Integral Adulto, Facultad de Odontología, Universidad de La Frontera, Temuco, Chile.
** División de Radiología Oral y Maxilofacial, Universidad de La Frontera, Temuco, Chile.

*** Kinesiologa, Temuco, Chile. 
gerado de la mandíbula hacia atrás (retrusión de la mandíbula) (Farag et al., 2012). A pesar de estas diferencias, las similitudes proporcionan ventajas significativas en el uso de los conejos en la investigación de la ATM (Mills et al.).

La tomografía computarizada cone-beam (TCCB) es un examen imagenológico menos costoso, con dosis de irradiación menor en comparación a la tomografía computarizada convencional. Además, proporciona imagen tridimensional del tejido mineralizado maxilofacial con un mínimo de distorsión. La TCCB es la técnica de imagen de elección para evaluar los componentes óseos de la ATM y suministra información esencial para ayudar en el diagnóstico de una variedad de alteraciones de la ATM, incluyendo osteoartritis, artritis inflamatoria, trauma y trastornos del desarrollo (Barghan et al., 2012).

El Láser de Baja Intensidad (LBI) es una de las modalidades de tratamiento muy utilizadas en el campo de la fisioterapia. El LBI produce un efecto bioestimulante, antiinflamatorio y analgésico a través de la irradiación directa sin provocar respuesta térmica (Snyder-Mackler \& Collender, 1996). En Odontología ha tenido amplia utilización y son muchas las especialidades odontológicas en las que se aplican las diferentes variedades de LBI. En el tratamiento de los trastornos temporomandibulares (TTMs) los resultados clínicos demuestran que el tratamiento por LBI es eficaz, siendo un método no invasivo indicado para el alivio del dolor agudo y especialmente del dolor miofascial crónico (Simunovic, 1996). Sin embargo, algunos investigadores señalan que el LBI presenta eficacia limitada en la reducción del dolor, no habiendo diferencias entre el grupo de pacientes con TTM tratados con LBI y el grupo placebo (Venancio et al., 2005; Emshoff et al., 2008; Chen et al., 2015).

La efectividad clínica, o sea, el éxito o fracaso de la terapia por LBI depende de la aplicación de una dosis de irradiación correcta (Simunovic). En el tratamiento de los TTMs los autores utilizan dosis de irradiación en un rango que va desde $3 \mathrm{~J} / \mathrm{cm}^{2}$ hasta $112,5 \mathrm{~J} / \mathrm{cm}^{2}$ por sesión de tratamiento (Emshoff et al.; Ferreira et al., 2013). En la literatura no hay ninguna evidencia precisa con respecto a la dosis efectiva para el tratamiento de los TTMs (Chen et al.).

El origen de evidencias científicas discordantes puede estar relacionada a la pluralidad de métodos de aplicación de irradiación láser no existiendo un protocolo indicando cuales son las dosis de irradiación, tiempo de tratamiento y la energía más eficaces y que no producen daños al paciente.
El objetivo de esta investigación fue estudiar los efectos de distintos protocolos de dosificación del LBI en el cóndilo mandibular de conejos, con el propósito de establecer cuales son las dosis de irradiación, utilizadas en este estudio, que producen alteraciones en las estructuras óseas detectables a través de TCCB.

\section{MATERIAL Y MÉTODO}

Este estudio fue aprovado por el Comité de Bioética de la Universidad de La Frontera (protocolo $n^{\circ}$ 074/13). Fueron utilizados 16 conejos de la raza Nueva Zelandia (Oryctolagos Cuniculos L.), adultos jóvenes, pesando entre 3 y $4 \mathrm{Kg}$. Los conejos fueron divididos aleatoriamente en cuatro grupos, $\mathrm{n}=4$ animales/grupo.

Examen de Tomografía Computadorizada Cone-Beam. Se realizó la toma de Tomografía Computarizada Cone-Beam (TCCB) en el Servicio de Imagenología de la clínica Odontológica de la Universidad de La Frontera, dicha adquisición fue realizada por la Radióloga del Servicio. Todos los exámenes imagenológicos tridimensionales fueron adquiridos con el Tomógrafo PAX Zenith 3D (Vatech Co., Gyeonggi-Do, Korea). Los datos obtenidos se exportaron en formato DICOM al software Ez3D 2009 (E-WOO Technology Co, Ltd. Korea).

Los exámenes fueron realizados con un Campo de $\mathrm{Vi}$ sualización o FOV de $12 \times 9 \mathrm{~cm}$, tamaño de voxel $0,12 \mathrm{~mm}$. Los parámetros de exposición utilizados son tiempo de exploración 24 s, 100 kv y 5 mA. La reconstrucción volumétrica en 3D fue realizada por el software Ez3D 2009. Se realizó un examen de TCCB inicial de ambas articulaciones de los 16 conejos, utilizados como grupo control (GC). Se realizó un segundo examen de TCCB, tras la irradiación por LBI, utilizado como grupo experimental (GE). Previo a los exámenes los animales fueron sedados con Ketamina $(0,5 \mathrm{~mL})$ y Xilacina $(0,6 \mathrm{~mL})$ vía intramuscular.

El análisis de las imágenes se hizo mediante la herramienta del software Ez3D 2009, siendo realizadas mediciones de cortes sagitales y coronales de ambas ATMs tomando el eje de las ramas mandibulares, en las imágenes de reconstrucción multiplanar.

Localización de eje mayor de ramas mandibulares: Determinada en el plano coronal de la Reconstrucción Multiplanar. Se obtiene una imagen bilateral de los cóndilos mandibulares (Fig.1).

Medición de cóndilos mandibulares en el corte coronal: Se midió usando la herramienta del software, se 


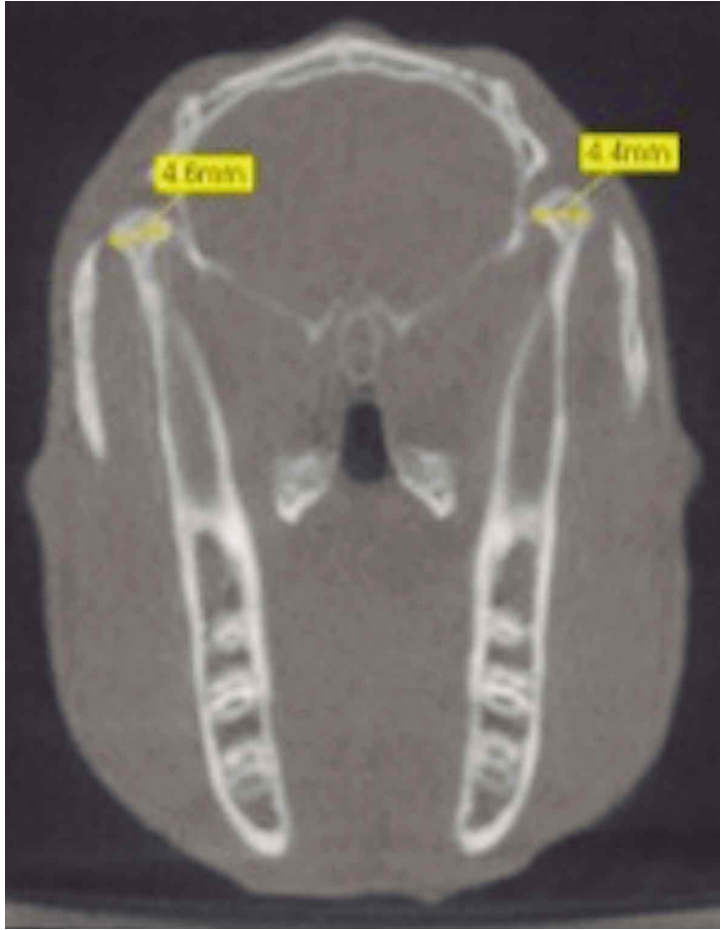

Fig. 1. Corte coronal, imagen bilateral de cóndilos mandibulares.

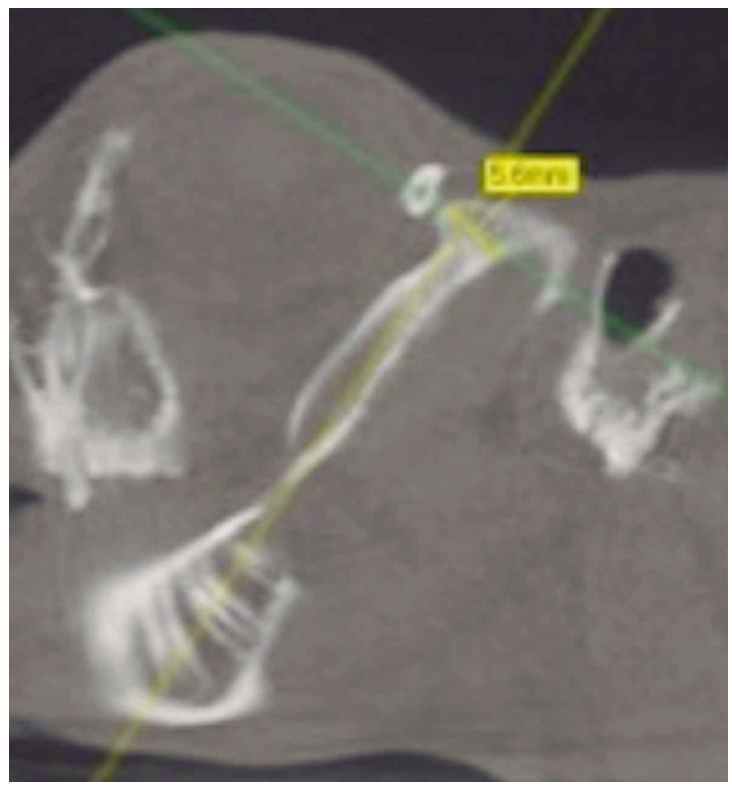

Fig. 2. Medición de cóndilos mandibulares en cortes sagitales, de acuerdo a eje de rama mandibular.

obtuvo la distancia entre el punto más proyectado hacia medial y el punto más proyectado hacia lateral en la cabeza de la mandíbula, expresada en milímetros (Fig. 1).
Medición de cóndilos mandibulares en el plano sagital: Utilizando la imagen coronal bilateral se determinó en forma individualizada el eje mayor de las ramas mandibulares para tener la imagen sagital. Posteriormente, se realizaron las mediciones considerando la distancia entre el punto más ventral y el punto más dorsal de la cabeza de la mandíbula (Fig. 2).

Protocolo de aplicación del Láser de Baja Intensidad. Para la aplicación del LBI se utilizó el As-Ga-Al 904 nm, 100 mW, spot $0,2 \mathrm{~cm}^{2}, 2$ puntos/ATM, 3 dosis/semana, totalizando 10 dosis. Los 16 animales fueron divididos en 4 grupos experimentales (GE) con 4 animales/grupo: GE 1 - $15 \mathrm{~J} / \mathrm{cm}^{2}, 30 \mathrm{~s}, 3 \mathrm{~J}$; GE 2- $45 \mathrm{~J} / \mathrm{cm}^{2}, 90 \mathrm{~s}, 9 \mathrm{~J}$; GE 3- $60 \mathrm{~J} / \mathrm{cm}^{2}, 120 \mathrm{~s}, 12 \mathrm{~J}$; GE 4- $90 \mathrm{~J} / \mathrm{cm}^{2}, 180 \mathrm{~s}, 18 \mathrm{~J}$. Tras 20 días de la última dosis de LBI ambas ATMs fueron evaluadas por la TCCB.

Para el análisis estadístico se utilizaron las pruebas de ANOVA y t-student para muestras correlacionadas, considerándose significativo $\mathrm{p}<0,05$.

\section{RESULTADOS}

En el análisis de las superficies articulares de los cóndilos mandibulares de conejos sometidos a LBI, no se ha observado alteraciones degenerativas detectables a través de TCCB.

Se ha observado alteraciones en las mediciones de los cóndilos mandibulares sometidos a LBI, a través de TCCB. Los valores promedios encontrados para los GEs fueron mayores que los valores encontrados para el GC en los cortes coronal y sagital (corte coronal: grupos 1, 2 y 3; corte sagital: grupos 1, 2, 3 y 4). No se encontró diferencia estadística significativa entre los grupos experimentales $1,2,3$ y 4 en los cortes sagital $(p=0,07)$ y coronal $(p=$ $0,48)$. En el corte coronal se encontró diferencia estadística significativa entre GC1 y GE1 ( $p=0,04), \operatorname{GC} 2$ y GE2 $(p=0,03)$. En el corte sagital no se encontró diferencia estadística significativa entre el grupo control y el grupo experimental (Tabla I).

Tabla I. Valores promedios, desviación estándar (DE) y valor p encontrados para los grupos control y experimental en cortes coronal y sagital.

\begin{tabular}{llcccc}
\hline & & \multicolumn{2}{c}{ Prome dio } & \multirow{2}{*}{ DE } & \multirow{2}{*}{ Valor $\boldsymbol{p}$} \\
\cline { 2 - 4 } Corte Coronal & & GC & GE & & \\
\cline { 2 - 4 } & Grupo 1 & 4,5 & 4,57 & $\pm 0,18$ & $0,04^{*}$ \\
& Grupo 2 & 4,08 & 4,13 & $\pm 0,43$ & $0,03^{*}$ \\
& Grupo 3 & 4,32 & 4,36 & $\pm 0,33$ & 0,28 \\
& Grupo 4 & 4,21 & 4,21 & $\pm 0,35$ & 0,39 \\
Corte Sagital & Grupo 1 & 5,0 & 5,15 & $\pm 0,43$ & 0,08 \\
& Grupo 2 & 4,86 & 5,31 & $\pm 0,65$ & 0,26 \\
& Grupo 3 & 4,87 & 4,9 & $\pm 0,40$ & 0,16 \\
& Grupo 4 & 5,16 & 5,17 & $\pm 0,45$ & 0,35 \\
\hline
\end{tabular}

GC= Grupo Control; GE= Grupo Experimental; *=diferencia estadística significativa. 


\section{DISCUSIÓN}

El conejo ha sido elegido en este estudio, porque su ATM presenta similitudes morfológicas con la ATM humana y también en relación a los movimientos mandibulares durante la masticación. Por lo tanto, es un modelo animal que proporciona ventajas significativas en la investigación con ATM (Mills et al.).

Hubo un tiempo en que se creía que la oclusión dental era la mayor responsable por las patologías de la ATM, los tratamientos eran poco conservadores e intervencionistas. Actualmente se sabe que los TTMs tienen una etiología controvertida y multifactorial y los tratamientos tienden a ser más conservadores y no invasivos, siendo que no hay un único estándar de tratamiento (Alves \& Cândido). Entre los recursos terapéuticos utilizados actualmente para el tratamiento de los TTMs está el LBI. El LBI y, en especial el As$\mathrm{Ga}-\mathrm{Al}$, se ha utilizado para el tratamiento de diversas enfermedades clínicas orofaciales, incluso en los TTMs.

Kulekcioglu et al. (2003) afirman que el uso del LBI en los TTMs es un buen método alternativo ya que reduce el dolor y aumenta la apertura oral y los movimientos de lateralidad. En esta misma línea Pinheiro et al. (1997, 1998) concluyeron que el uso del LBI es un método efectivo y beneficioso en el tratamiento de gran cantidad de alteraciones de la región maxilofacial como el dolor articular, la neuralgia del trigémino y el dolor muscular entre otros.

A pesar de la constante evolución y desarrollo en la investigación de esta tecnología, el mecanismo de acción implicado en el tratamiento y el efecto específico en la ATM, todavía son poco claros.

En nuestro estudio no se ha observado alteraciones degenerativas en las superficies articulares de los cóndilos mandibulares sometidos a LBI, a través de TCCB, sin embargo observamos alteraciones en sus medidas. De la misma manera Matamala et al. (2009) evaluando el efecto del láser As-Ga, $780 \mathrm{~nm}$ sobre ATM de conejos encontraron aumento de los diámetros anteroposterior y transversal del cóndilo mandibular. Creemos que la dosimetría (o fluencia) puede tener influencia en los cambios morfológicos observados en los tejidos irradiados.

Es indiscutible la importancia del conocimiento de la cantidad de energía que se utiliza en un determinado tratamiento para lograr el efecto deseado. En relación a la dosis de radiación, existe gran diversidad de criterios respecto los parámetros de energía, potencia y tiempo que se debe utilizar. De acuerdo con algunos investigadores, dosis bajas producen un efecto de estimulación del metabolismo celular mientras que dosis altas producen efectos dañinos a la célula (Amaral et al., 2001; Houghton \& Brown, 2000).

Hawkins \& Abrahamse (2006) señalan que la dosimetría (o fluencia) puede cambiar los procesos celulares. La aplicación de una dosis baja o muy baja puede producir ningún efecto o incluso efectos negativos o inhibidores. Eso podría explicar los resultados antagónicos encontrados en las investigaciones (Simunovic).

Otros autores, consideran que la potencia empleada por unidad de área $\left(\mathrm{W} / \mathrm{m}^{2}\right)$, también llamada densidad de potencia es el factor más importante para lograr el efecto terapéutico deseado.

Según Valiente \& Garrigó (2006) para lograr el efecto terapéutico deseado es importante establecer la frecuencia de irradiación, pudiendo irradiarse diariamente o en días alternos, dependiendo de la enfermedad a tratar, sea un proceso agudo o crónico, presencia de dolor, severidad de la lesión e incluso experiencia personal, según resultados obtenidos. Estas autoras afirman aún que en Estomatología, por lo general, el número de sesiones a programar no son más de 10 y recomiendan una o dos aplicaciones más después de eliminado el dolor o curada la lesión.

Creemos que el clínico debe tomar en cuenta varios aspectos para calcular la dosis, tales como edad del paciente, su condición sistémica, tipo de patología a tratar, profundidad, además de otras características específicas obtenidas mediante examen clínico. Además, es muy importante estar siempre atento no solamente a los efectos deseados sino también a los efectos adversos.

Es importante recalcar que en la literatura los efectos adversos del LBI no están descritos de forma precisa, tampoco su inocuidad. Considerando que existen pocos estudios a respecto, creemos que existe una clara necesidad de investigar sus efectos mediante estudios experimentales en modelo animal.

En base a los resultados de nuestro estudio concluimos que los valores promedios encontrados en las mediciones de los cóndilos mandibulares, sometidos a las dosis de LBI utilizadas en este estudio (GE), fueron mayores que los valores promedios encontrados para el GC en planos coronal y sagital. Además, podemos afirmar que la TCCB demostró ser un método eficaz para el análisis de las dimensiones condilares.

AGRADECIMIENTOS. Este trabajo fue apoyado por Dirección de Investigación, Universidad de La Frontera, Projecto DI14-0043. 
ALVES, N.; GARAY, I. \& DEANA, N. F. Effects of low-level laser (Ga-Al-As $904 \mathrm{~nm}$ ) on the mandibular condyle of rabbits: analysis by cone beam computed tomography. Int. J. Morphol., 34(1):218-222, 2016.

SUMMARY: In the scientific literature the adverse effects of Low Level Laser (LLL) are not precisely described, neither is their safety. The aim of this study was to evaluate the effect of different protocol doses of laser on the mandibular condyle of rabbits. Sixteen rabbits randomly divided into four experimental groups (EG) of 4 animals were used in this study. We carried out a cone beam computed tomography (CBCT) examination of both temporomandibular joints (TMJs) of all animals which were used as control. Subsequently, we radiated each TMJ with LLL (Ga-Al-As $904 \mathrm{~nm}$ ), 100mW, spot 0.2 $\mathrm{cm}^{2}$, two points, 3 times/week, totaling 10 times, with doses of $15 \mathrm{~J} /$ $\mathrm{cm}^{2}$ (EG-1), $45 \mathrm{~J} / \mathrm{cm}^{2}$ (EG-2), $60 \mathrm{~J} / \mathrm{cm}^{2}$ (EG-3) and $90 \mathrm{~J} / \mathrm{cm}^{2}$ (EG-4). After 20 days of the last irradiation we performed a new CBCT exam and then the measurement of the condyles on the coronal and sagittal planes was obtained. In the analysis of the results ANOVA and t-student for correlated samples was used. The average values found for the EG were higher than those found for CG for both the coronal and sagittal planes. There was a significant statistical difference for group 1 and between the groups 1 and 2 . We concluded that the average values found in the measurements of the mandibular condyles, submitted to doses of LLL used in this study, were higher than those found for the control group in both the coronal and sagittal planes. Furthermore, the CBCT proved to be an effective method for analysis of condylar dimensions.

KEY WORDS: Cone-beam computed tomography; Temporomandibular joint; Rabbit; Animal model; Low-level laser.

\section{REFERENCIAS BIBLIOGRÁFICAS}

Alves, N. \& Cândido, P. L. Anatomia para o Cirurgião-Dentista. $2^{\mathrm{a}}$ ed. São Paulo, Gen-Santos, 2013.

Amaral, A. C.; Parizotto, N. A. \& Salvini, T. F. Dose-dependency of lowenergy HeNe laser effect in regeneration of skeletal muscle in mice. Lasers Med. Sci., 16(1):44-51, 2001.

Barghan, S.; Tetradis, S \& Mallya, S. Application of cone beam computed tomography for assessment of the temporomandibular joints. Aust. Dent. J., 57 (Suppl. 1):109-18, 2012.

Chen, J.; Huang, Z.; Ge, M. \& Gao, M. Efficacy of low-level laser therapy in the treatment of TMDs: a meta-analysis of 14 randomised controlled trials. J. Oral Rehabil., 42(4):291-9, 2015.

Emshoff, R.; Bösch, R.; Pümpel, E.; Schöning, H. \& Strobl, H. Low-level laser therapy for treatment of temporomandibular joint pain: a doubleblind and placebo-controlled trial. Oral Surg. Oral Med. Oral Pathol. Oral Radiol. Endod., 105(4):452-6, 2008.

Farag, F. M.; Daghash, S. M.; Mohamed, E. F.; Hussein, M. M. \& Hagrass, S. M. Anatomical studies on the skull of the domestic rabbit (Oryctolagus cuniculus) with special reference to the hyoid apparatus. J. Vet. Anat., 5(2):49-70, 2012

Ferreira, L. A.; de Oliveira, R. G.; Guimarães, J. P.; Carvalho, A. C. \& De Paula, M. V. Laser acupuncture in patients with temporomandibular dysfunction: a randomized controlled trial. Lasers Med. Sci., 28(6):1549-58, 2013

Hawkins, D. H. \& Abrahamse, H. The role of laser fluence in cell viability, proliferation, and membrane integrity of wounded human skin fibroblasts following helium-neon laser irradiation. Lasers Surg. Med., 38(1):74-83, 2006.

Houghton, P. E. \& Brown, J. L. Effect of low level laser on healing in wounded fetal mouse limbs. Laser Ther, 11(2):54-68, 2000.

Kulekcioglu, S.; Sivrioglu, K; Ozcan, O. \& Parlak, M. Effectiveness of lowlevel laser therapy in temporomandibular disorder. Scand. J. Rheumatol., 32(2):114-8, 2003.

Matamala, V. F.; Cornejo, U. R.; Vasconcellos, C. A. \& Silva, M. H. Efecto del láser asga sobre la anatomía normal de la articulación témporo mandibular (ATM) de conejos. Rev. Cient. (Maracaibo), 19(1):31-7, 2009.

Mills, D. K.; Daniel, J. C.; Herzog, S. \& Scapino, R. P. An animal model for studying mechanisms in human temporomandibular joint disc derangement. J. Oral Maxillofac. Surg., 52(12):1279-92, 1994.

Pinheiro, A. L.; Cavalcanti, E. T.; Pinheiro, T. I.; Alves, M. J. \& Manzi, C. T. Low-level laser therapy in the management of disorders of the maxillofacial region. J. Clin. Laser Med. Surg., 15(4):181-3, 1997.

Pinheiro, A. L.; Cavalcanti, E. T.; Pinheiro, T. I.; Alves, M. J.; Miranda, E. R.; De Quevedo, A. S.; Manzi, C. T.; Vieira, A. L. \& Rolim, A. B. Low-level laser therapy is an important tool to treat disorders of the maxillofacial region. J. Clin. Laser Med. Surg., 16(4):223-6, 1998.

Simunovic, Z. Low level laser therapy with trigger points technique: a clinical study on 243 patients. J. Clin. Laser Med. Surg., 14(4):163-7, 1996.

Snyder-Mackler, L. \& Collender, S. L. Therapeutic uses of light in rehabilitation. In: Michlovitz, S. L. Thermal Agents in Rehabilitation. Contemporary Perspectives in Rehabilitation. $3^{\text {rd }}$ ed. Philadelphia, F.A. Davis, 1996.

Valiente Zaldívar, C. \& Garrigó Andreu, M. I. Laserterapia y Laserpuntura para Estomatología. La Habana, Editorial Ciencias Médicas, 2006.

Venancio, Rde. A.; Camparis, C. M. \& Lizarelli, Rde. F. Low intensity laser therapy in the treatment of temporomandibular disorders: a double-blind study. J. Oral Rehabil., 32(11):800-7, 2005.

\section{Dirección para Correspondencia:}

Nilton Alves

Departamento de Odontología Integral Adulto,

Facultad de Odontología

Universidad de La Frontera

Avenida Francisco Salazar, 1145

Casilla 54-D.

Temuco

CHILE

\section{Email: niltonnalves@yahoo.com.br}

Recibido : 28-07-2015

Aceptado: 17-11-2015 